\title{
CARING FOR OLDER ADULTS WITH MENTAL HEALTH DISORDERS DURING THE PANDEMIC
}

Ms. Neena Mathew*

*MSN, FNP-BC, PMHNP-BC

\section{ABSTRACT}

DOI: http://doi.org/10.47211/trr.2021.v07i02.008

\begin{abstract}
Older adults (65 years or older) are prone to various mental health disorders, which require appropriate care. Mental illnesses like depression, dementia and Alzheimer's disease, among others, increase cognitive deterioration, which leads to dependence on normal activities in life. During pandemics like COVID-19 and Ebola, the mentally ill older adults are highly susceptible to increased morbidity, mortality rates and reduced quality and social life hence require appropriate care. Pandemic occurrence is a concern and worry for the mentally ill older adults since they have an increased vulnerability and are highly dependent on help and care. Moreover, a pandemic like COVID-19 may lead to neurological and mental health complications, which may adversely affect the mentally ill older adults. During the initial stages of the COVID-19 pandemic, older adults were more likely to be infected with the virus and have worse outcomes than their younger counterparts. Similarly, morbidity and mortality rates were highest among older adults who had co-occurring conditions such as hypertension, cardiovascular disease, diabetes, chronic respiratory disease, and chronic kidney disease. Older adults were also susceptible to loneliness and social exclusion. The risk factors that were associated with worsening of physical, psychological, and social well-being among these individuals included age, ageism, isolation, loneliness, lack of security, frailty dependency, sexism, stigma, abuse, and cognitive and sensory impairments. The pandemic also had a significant effect on the mental health of older adults.
\end{abstract}

Key words: Corona Virus Disease (COVID-19), psychological responses, geographical locations.

\section{ABOUT AUTHOR:}

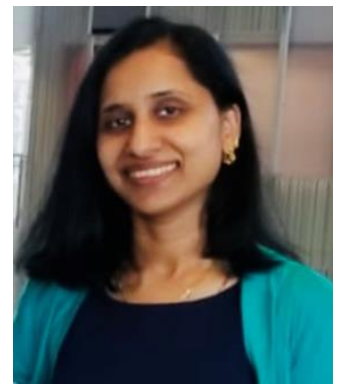

Author Ms. Mathew is a Board Certified Nurse Practitioner who is qualified to practice in both primary care and psychiatry; diagnose, treat, and prescribe medication for across the life span including children, adolescents, adults and older adults. She is currently working as a Lead Nurse Practitioner with Park Place Behavioral Healthcare, since 2016, which is a community mental health care facility in Orlando Florida. Some of her practice specialties include (but not limited to) ADHD, Depression, Anxiety and Bipolar. Mathew's style of practice can be traditional (prescribing up to date psychiatric medications) or alternative medicine (natural treatments including but not limited to vitamins, supplements, lifestyle modification, nutrition, exercise and stress management). She believes in the mind/body/spiritual connection, and we are made up as a whole person and not a series of parts, just as Hippocrates "The Father of Medicine." 


\section{ARTICLES}

\section{INTRODUCTION}

The population of older adults in the United States is increasing rapidly. It is estimated that their population will nearly double from 15\% in 2014 to $24 \%$ (98 million) of the total population by 2060. Currently, 1 in 5 older adults in the United States has a diagnosable mental health disorder, with personality disorders, anxiety disorders, mood disorders, and substance use disorders being the most common. It is also estimated that the total of individuals with Alzheimer disease in the United States will rise from 5 million in 2013 to approximately 14 million by 2050. Caring for older adults is essential (Alves et al., 2020) since individuals desire and deserve a life, with good health, dignity, economic independence, and improved quality of life. Since they are vulnerable populations, the older adults are prone to mental illness (Cremonini et al., 2018) that affects how they perform their daily activities. The individuals are affected during the pandemic, increasing their dependency levels. Previous researches by Kelly et al. (2017) mention that it is essential to comprehend the needs and concerns of older adults to ensure good health and improved quality of life. Pandemics are associated with various health consequences depriving individuals of their well-being (Van Tilburg et al., 2021), increased risks of neglect, abuse, helpless and are ignored. Therefore, the older adults need a lot of assistance and monitoring during such situations achieved by providing support in healthcare, emotional, psychological, and physical aspects of care. COVID-19 infection presented as altered mental status in the absence of respiratory symptoms or fever. Worsening of confusion, agitation, disorientation, refusing care, and apathy were also presenting symptoms among this population. Older adults also reported greater rates of loneliness, depression, risk for substance use disorders, and suicide. In addition, worsening of psychiatric symptoms was noted among older adults who were admitted to hospitals during the lockdown periods. Pandemic situations are characterized by threatening and challenging moments that affect vulnerable older adults' wellbeing (Ester wood \& Saeed, 2020). Therefore, caring for the geriatrics faced with pandemics is achieved by providing nutritious foods, basic supplies, financial help, social care and medicine to support their physical health. The families' support provides an opportunity for embracing some form of love and kindness to the older adults with cognitive illnesses.

Huremović (2019) supports that caring for older adults during pandemics is essential for a comprehensive response to pandemics. Support effectively ensures that older adults have clear messages and resources on how to stay physically and mentally healthy during the pandemic and the remedies if they fall ill (Magill, Siegel and Pike, 2020). Since the older adults are vulnerable to illness which may occur as pandemics leading to quarantine and isolation, care can be embraced through providing protection devices, good ventilated rooms, with provision for separate rooms. Caring can also be ensured through providing education on maintenance of hygiene, safety, and settings to minimize the risk of transmissions.

Caring for older adults is the duty (Mukku \& Sivakumar, 2020) of the family members and the individuals within the environment to ensure they are safe. Caring for them generally involves monitoring their care at home, enabling social inclusion (National Academies of Sciences, Engineering, and Medicine. 2020), ensuring that the individuals' treatments are followed up, and learning and practicing mindful communication. In some instances of the pandemic, the older adults may find coping with their mental illness a challenge. To care for older adults with mental health disorders during the pandemic and to minimize the effects of sustained social isolation, many alternative methods to providing care were developed. Electronic devices or platforms to conduct virtual interactions for social, profession, or clinical interactions became important tools. Telephone evaluations were conducted for older adults who were not able to use technology. In addition, community connection websites were established to provide a searchable directory of organizations that provided essential daily services for older adults. Older adults were also encouraged to participate in social, spiritual, or religious interactions using electronic means/platforms. Clinicians who shifted to telepsychiatry in 2020 and 2021 reported high overall satisfaction with remote care, according to a study in the Journal of the Academy of Consultation-Liaison Psychiatry. Care support can be provided to educate them on how to cope with (Neelam et al., 2021) changes within the environment and the appropriate actions they should take to deal with life challenges brought about by the pandemic. In the United States, older adults occupy the highest proportion of population as compared to the other age groups. The country is therefore more prone to elderly with mental disorders (Satre et al., 2021) and is among the affected with an increased number of incidences of COVID-19. The individuals require care since they are highly susceptible to infections; hence (Pain and Lanius, 2020), they may be faced with significant risks of contracting illness during pandemic since they have cognitive illnesses. The illness may lead to contracting the 


\section{ARTICLES}

disease due to physiological changes that come with ageing and mental illness because of the underlying comorbid conditions.

Healthcare providers and families find challenges in caring for the mentally ill older adults during pandemics since most of them have cognitive impairments which worsen their behavioral problems. The victims are faced with challenges like low-income family support, financial constraints, and increased dependency. Older adults require supportive care at this particular period (Neelam et al., 2021) since they cannot manage their situations alone. Care is an important way of reducing the combined burden of mental illness and pandemics. In some situations, pandemics could be in the form of fire outbreaks and disasters like floods and earthquakes, which worsen the victims' health and quality of life.

Individuals require material, emotional, mental, and psychological support during such critical moments. The burden of the illness comes with particular symptoms like sleep disturbances, feeling, dependency (Mukku \& Sivakumar, 2020), anxiety, and depression. Pandemic situations increase the negative health consequences to their health. They need family support to prevent loneliness and improve behavioral actions that enhance the overall quality of life. Older adults have increased energy needs during pandemics. Therefore, they require nutritional support to improve their mental health condition. Since mental illness manifests with sleep disturbances and increased risk for delirium, older adults need support from the family and community regarding transport, protection from harm from the calamity, and traveling long distances. The families help keep the older adults from strong emotional reactions to future experiences from natural disasters (Esterwood \& Saeed, 2020). Support in terms of care ensures a higher resilience and sound support system to overcome the ongoing pandemic through other forms of support such as wisdom and learning from previous disasters experiences.

The community can embrace care for the older individuals by offering protection from diseases like COVID-19 by ensuring they are vaccinated and isolated in safe places free from the virus. The infected older adults undergoing isolation can be supported both physically and emotionally to keep them off the stress of being lonely and pressure that may emerge in them. Despite the caregivers and healthcare providers providing support to the older adults, they encounter physical and mental stress since the victims have increased demands. However, it is the duty of the families and (Esterwood \& Saeed, 2020) the community to ensure the older adults are given assistance like wheelchairs, finances, foodstuffs and are helped in seeking prompt treatments when encountering such situations.

Moreover, support may be informed of spending quality time with them and having meaningful conversations concerning instilling joy and hope in them. They can be kept involved by engaging them about memories from the past and helping them stay active and occupied. Safety is to be maintained in terms of health, services for adults, and vulnerability to the pandemic.

\section{CONCLUSION}

Mental health issues increase the concern among older adults due to vulnerability and increased burden. During pandemics like disease occurrence and natural calamities, the vulnerable populations such as the older adults, remain hopeless, helpless, which in turn may worsen their behavioral problems. Therefore, older adults with mental illness should be cared for during pandemics since they are highly susceptible to mortality and morbidities. Support provided through emotional, physical, psychological and economic perspectives may be of help. Despite the difficulties they endured during the pandemic, older adults with mental health disorders in the United States have done fairly well, compared to younger adults. Families, friends, clinicians, social support systems, health care agencies, health care systems, and governmental and nongovernmental agencies have rallied together during this period of uncertainty to ensure that the provision of care to these vulnerable individuals did not suffer significantly.

It would call for responsibility from us as clinicians, to use the lessons learned during the pandemic to continue to improve the psychiatric care of older adults going forward. By doing so, we can enhance the quality of life of these individuals and minimize the negative effects any such future global events may have on their lives. 


\section{ARTICLES}

\section{REFERENCES}

1. Alves, G. S., Casali, M. E., Veras, A. B., Carrilho, C. G., Bruno Costa, E., Rodrigues, V. M., \& Dourado, M. C. N. (2020). A systematic review of home-setting psychoeducation interventions for behavioral changes in dementia: some lessons for the COVID-19 pandemic and post-pandemic assistance. Frontiers in psychiatry, 11, 1028.

2. Cremonini, V., Pagnucci, N., Giacometti, F., \& Rubbi, I. (2018). Health care professionals attitudes towards mental illness: Observational study performed at a public health facility in northern Italy. Archives of Psychiatric Nursing, 32(1), 24-30.

3. Esterwood, E., \& Saeed, S. A. (2020). Past epidemics, natural disasters, COVID19, and mental health: learning from history as we deal with the present and prepare for the future. Psychiatric quarterly, 1-13.

4. Huremović, D. (Ed.). (2019). Psychiatry of pandemics: a mental health response to infection outbreak. Springer.

5. Kelly, M. E., Duff, H., Kelly, S., Power, J. E. M., Brennan, S., Lawlor, B. A., \& Loughrey, D. G. (2017). The impact of social activities, social networks, social support and social relationships on the cognitive functioning of healthy older adults: a systematic review. Systematic reviews, 6(1), 1-18.

6. Magill, E., Siegel, Z., \& Pike, K. M. (2020). The mental health of frontline health care providers during pandemics: a rapid review of the literature. Psychiatric Services, 71(12), 1260-1269.

7. Mukku, S., \& Sivakumar, P. (2020). Mental Health Care of Older Adults during Coronavirus Disease (COVID-19) Pandemic: Challenges and Way ahead in India. Indian journal of psychological medicine, 42(5), 495-496. https://doi.org/10.1177/0253717620944824

8. National Academies of Sciences, Engineering, and Medicine. (2020). Social isolation and loneliness in older adults: Opportunities for the health care system. National Academies Press.

9. Neelam, K., Duddu, V., Anyim, N., Neelam, J., \& Lewis, S. (2021). Pandemics and pre-existing mental illness: A systematic review and meta-analysis. Brain, behavior, \& immunity-health, 10, 100177.

10. Pain, C., \& Lanius, R. (2020). Disasters, pandemics and mental health. CMAJ, 192(28), E803-E803.

11. Satre, D. D., Hirschtritt, M. E., Silverberg, M. J., \& Sterling, S. A. (2020). Addressing problems with alcohol and other substances among older adults during the COVID-19 pandemic. The American Journal of Geriatric Psychiatry, 28(7), 780-783.

12. Van Tilburg, T. G., Steinmetz, S., Stolte, E., van der Roest, H., \& de Vries, D. H. (2021). Loneliness and mental health during the COVID-19 pandemic: A study among Dutch older adults. The Journals of Gerontology: Series B, 76(7), e249-e255. 